\title{
Marcação de Diatraea saccharalis (Fabr.) com Diferentes Corantes em Dieta Artificial
}

\author{
Jael Simões Santos Rando ${ }^{\bowtie}$, Laila Herta Mihsfeldt, Franciele Paulette Lial de Souza \& Fernanda Venancio Soares
}

Universidade Estadual do Norte do Paraná / Campus Luiz Meneghel, e-mail: jael@uenp.edu.br (Autor para correspondência ${ }^{\bowtie}$ ), lailaherta@uenp.edu.br, franplsouza@gmail.com.

\section{EntomoBrasilis 9 (1): 47-5o (2016)}

\begin{abstract}
Resumo. Para estudar a distribuição espacial da broca da cana-de-açúcar Diatraea saccharalis (Fabr.) e seu parasitoide Cotesia flavipes (Cameron) no campo, o uso de marcadores tem se mostrado promissor. Estes marcadores devem permanecer no inseto até o momento de recaptura, não podendo afetar o ciclo biológico. Este estudo avaliou o efeito de corantes como marcadores na dieta de alimentação da broca da cana-de-açúcar. Os insetos foram provenientes do laboratório de criação da USIBAN (Bandeirantes-PR). Utilizou-se dieta de alimentação usada na rotina do laboratório acrescida de: açafrão $(10 \mathrm{~g})$, corante azul brilhante $(2 \mathrm{~mL})$, violeta genciana $(2 \mathrm{~mL})$, iodo $(2 \mathrm{~mL})$, urucum $(10 \mathrm{~g})$, e como testemunha a dieta sem corante. Foram preparados 40 tubos/dieta e cada um recebeu dez lagartas recém-eclodidas. O material foi mantido em estuda BOD a $25^{\circ} \mathrm{C}$ e avaliou-se: duração dos períodos larval e pupal, viabilidades larval e pupal. As pupas foram separadas por sexo e um casal de adultos liberados em gaiolas de PVC forradas com papel sulfite, totalizando dez casais por tratamento. Avaliou-se longevidade dos adultos e número de ovos. O iodo foi tóxico para as lagartas. Os outros corantes apresentaram prolongamento do período larval, mas sem diferir estatisticamente da testemunha. O período pupal não foi afetado pela adição dos corantes. No urucum não houve número suficiente de adultos; este corante e o azul brilhante apresentaram prolongamento na longevidade dos adultos. O número total de ovos inferior ao esperado e a viabilidade total inferior a $75 \%$ em todos os tratamentos. Sugere-se a continuidade dos estudos para obtenção de outras técnicas de marcação.
\end{abstract}

Palavras-chave: Controle biológico; biologia; criação massal; Saccharum spp.; broca da cana-de-açúcar.

\section{Marking of Diatraea saccharalis (Fabr.) with Dyes in Artificial Diet}

Abstract. To study the spatial distribution of drill sugarcane Diatraea saccharalis (Fabr.) and its parasitoid Cotesia flavipes (Cameron) in the field, the use of markers has shown promise. These markers should remain in the insect so far recapture and can not affect the biological cycle. This study evaluated the effect of dyes as markers on the drill feed diet sugarcane. The insects were from the laboratory creation of USIBAN (BandeirantesPR). Feeding diet was used used in the routine of increased laboratory: Saffron (10g) brilliant blue dye (2 mL), gentian violet (2 mL), iodine ( $2 \mathrm{~mL})$, "urucum" (10g) and as a control diet without dye. They were prepared 40 tubes / diet and each received ten newly hatched caterpillars. The material was kept studying chamber at $25{ }^{\circ} \mathrm{C}$ and were evaluated: duration of larval and pupal periods, larval and pupal viability. The pupae were separated by sex and a couple of adults released in PVC cages lined with bond paper, totaling ten couples per treatment. It evaluated longevity number of adults and eggs. Iodine is toxic to the caterpillars. The other dyes showed prolonged larval period, but not statistically differ from the control. The pupal period was not affected by the addition of dyes. In "urucum" was not enough adults; this dye and bright blue had extended the longevity of adults. The total number of eggs lower than expected and the overall viability below $75 \%$ in all treatments. It is suggested to continue their studies to obtain other labeling techniques.

Keywords: Biological control; biology; mass rearing; Saccharum spp.; sugarcane borer.

ocorrência de Diatraea saccharalis (Fabr.) (Lepidoptera: Cambridae), conhecida como broca da cana-de-açúcar, é uma das adversidades enfrentadas pela cultura canavieira, pois ocorre praticamente durante todo o período de desenvolvimento. Para seu controle, vem sendo empregada a vespa Cotesia flavipes (Cameron) (Hymenoptera: Braconidae). O parasitoide é produzido em larga escala em laboratórios de vários países, inclusive no Brasil, e suas liberações têm mostrado que ele é um eficiente regulador da população praga no campo (Pinto et al 2006).

A criação de insetos em dietas artificiais tem gerado avanços nas práticas de manejo integrado de pragas (KING 1975). Sua utilização tem como vantagens a obtenção contínua de indivíduos com uniformidade nutricional e biológica, controle mais eficiente de patógenos e possibilidade de automação para eventual criação massal (PARRA 2002). No Brasil, a produção massal de C. flavipes é diferente entre biofábricas, podendo ocorrer modificações nos componentes usados na dita artificial empregada na alimentação do hospedeiro $D$. saccharalis com o objetivo de diminuir o custo de produção, porém sem critérios científicos (VEIGA et al. 2013).
A criação massal de insetos requer controle de qualidade quanto as gerações produzidas em laboratório. Os parasitoides produzidos em laboratório são utilizados em estudos de distribuição, os quais são vitais para entender sua área de cobertura após liberação.estando relacionadas com estudos de distribuição de um parasitoide após sua liberação (CASTILLo et al. 2006).

A marcação de insetos pode ser utilizada como artifício em pesquisas que visem a obtenção de informações sobre amostragem absoluta de insetos (Southwood 1978). Nesse contexto, o uso de corantes para marcação é de fácil aplicação e visualização, possibilitando marcação permanente; devem ser de fácil manipulação e seguros ao ambiente (HAGLER \& JACKSON 2001). Em estudos de liberação e captura, os insetos marcados ficam facilmente distintos dos insetos presentes naturalmente no campo, garantindo a marcação do inseto até o momento de recaptura (SOUTHWOOD 1992). Os corantes, assim como qualquer técnica de marcação não podem afetar o desenvolvimento do

Agência de Financiamento: Fundação Araucária 
T inseto, sua probabilidade de captura, devem ser duráveis, sem afetar longevidade e comportamento (FERNANDES 2002). A busca por técnicas e produtos de baixo custo é uma das bases em programas de controle biológico.

A marcação interna de insetos costuma ser feita com Calco Oil Red (COR), Rodamina B, sulfato azul do Nilo, oxido crômico, tintas de carimbo, etc. Na criação de $D$. saccharalis em dieta artificial com Calco Oil Red, os adultos de Cotesia flavipes que nela se desenvolveram tornaram-se coloridos (FERNANDES 2002). Os corantes Sudan Red 7B e Solvent Blue marcaram adultos e ovos de $D$. saccharalis, porém somente o corante Sudan Red $7 \mathrm{~B}$ na concentração de 100 ppm não afetou sua biologia (VILARINHO 2007). Volpe (2009) utilizou o corante Sudan Red B e relatou prolongamento da fase larval em $D$. saccharalis. Nos corantes naturais existe a dificuldade em extrair e manter a coloração em todo o período de desenvolvimento do inseto, como relatado por Souza et al (2013), utilizando beterraba "in natura".

Com base nessas informações, o objetivo do trabalho foi verificar o efeito de corantes sobre a biologia de $D$. saccharalis em dieta artificial.

\section{MATERIAIS E MÉTODOS}

Os insetos foram provenientes de criação mantida do laboratório da USIBAN - Açúcar e Álcool Bandeirantes S/A (Bandeirantes - PR). Os adultos de D. saccharalis (20 fêmeas e 30 machos) foram mantidos em gaiolas de acasalamento (tubos de PVC de $20 \mathrm{~cm}$ de altura por $10 \mathrm{~cm}$ diâmetro), forradas com papel sulfite, para obtenção dos ovos. As folhas com posturas foram retiradas das gaiolas e receberam tratamento de desinfecção externa, através da imersão das mesmas em solução de sulfato de cobre a 1\% por um minuto, enxague em água destilada e secagem. Após, as posturas foram recortadas e colocadas em placas de Petri contendo papel filtro umedecido com água.

Para a alimentação das lagartas de $D$. saccharalis foi utilizada dieta artificial (Tabela 1), com cinco corantes (tratamentos) e sem corante (testemunha) (Tabela 2).

Tabela 1. Composição da dieta de alimentação de Diatraea saccharalis usada na rotina do laboratório da USIBAN. Bandeirantes-PR, 2015.

\begin{tabular}{lc}
\hline \multicolumn{1}{c}{ Ingredientes } & Quantidade \\
\hline Açúcar & $280 \mathrm{~g}$ \\
\hline Farelo de soja & $300 \mathrm{~g}$ \\
\hline Germe de trigo & $200 \mathrm{~g}$ \\
\hline Nipagin & $22 \mathrm{~g}$ \\
\hline Ácido ascórbico & $10 \mathrm{~g}$ \\
\hline Cloreto de colina & $2 \mathrm{~g}$ \\
\hline Sais de Wesson & $20 \mathrm{~g}$ \\
\hline Solução vitamínica & $30 \mathrm{~mL}$ \\
\hline Tetraciclina & 2 cápsulas \\
Formaldeído & $5 \mathrm{~mL}$ \\
\hline Glicopan & $2 \mathrm{~mL}$ \\
\hline Caraginato & $70 \mathrm{~g}$ \\
\hline Água (liquidificador) & $2000 \mathrm{~mL}$ \\
\hline Água (caraginato) & $2000 \mathrm{~mL}$ \\
\hline
\end{tabular}

As dietas foram preparadas de acordo com Parra (2001), e transferidas para tubos de fundo chato tampados com algodão hidrofóbico após esterilização a seco em estufa a $180^{\circ} \mathrm{C}$, por duas horas. Utilizaram se 40 tubos por tratamento, e cada um recebeu dez lagartas recém-eclodidas. O material foi mantido em estufa do tipo $\mathrm{BOD}$ a $25^{\circ} \mathrm{C}$ durante o período de desenvolvimento larval e pupal, com observações diárias. Foram avaliados a duração e viabilidade dos períodos larval e pupal. As pupas foram separadas por sexo. Em cada uma das dez gaiolas por tratamento foi liberado um casal, avaliando-se a longevidade e o número total de ovos por fêmea.

Tabela 2. Identificação dos tratamentos usados para avaliar o efeito de corantes adicionados a dieta de alimentação de Diatraea saccharalis. Bandeirantes-PR, 2015.

\section{Tratamento}

\begin{tabular}{ll} 
A & Testemunha (sem adição de corante) \\
B & Dieta + 10 g de açafrão \\
C & Dieta + 2 mL de corante azul brilhante \\
D & Dieta + 2 mL de corante violeta genciana \\
E & Dieta + 2 mL de iodo \\
F & Dieta + 10 g urucum \\
\hline
\end{tabular}

Os dados obtidos foram submetidos à análise de variância (ANOVA), e Teste de Tukey a 5\% de probabilidade quando a ANOVA indicou diferenças significativas. Os cálculos estatísticos foram realizados pelo programa Statistica versão 8.o (STATSofT 2007).

\section{RESULTADOS E DISCUSSÃO}

O iodo teve efeito tóxico sobre $D$. saccharalis. Os outros corantes apresentaram prolongamento do período larval, embora não tenham diferenciado estatisticamente da testemunha (Tabela 3). Os dados vão de encontro aos obtidos por PARRA \& MiHSFELDT (1992), que em dietas sem adição de corantes obtiveram duração média foi em torno de 30 dias. Ao utilizar beterraba in natura como corante, SouzA et al. (2013) relataram que a duração do período larval não foi alterada no tratamento que recebeu menor concentração do corante natural; porém, a marcação das lagartas não ocorreu em nenhuma das concentrações utilizadas. A marcação de D. saccharalis ocorreu com o corante artificial Vermelho de Sudan B (VolPe 2009), porém, houve prolongamento da duração do período larval.

A duração do período pupal não apresentou diferenças significativas entre os tratamentos (Tabela 3), não sendo afetado pela adição dos corantes. Estes valores corroboram com PARRA \& Minsfeldt (1992), GAllo et al. (2002) e Volpe (2009), os quais citam que as pupas podem permanecer nesta fase por 9 a 14 dias.

Os adultos apresentaram maior longevidade nas dietas acrescidas com azul brilhante e urucum (Tabela 3). Tais valores são discrepantes dos relatados por BotelHo (1985), MENDONÇA (1996), GALlo et al. (2002) e VolPE (2009), os quais relataram esta fase em torno de cinco dias. Porém Lima (2011), ao trabalhar com a dieta de Hensley \& Hammond (1968) com modificações na sua composição, relatou longevidade superior a 9 dias.

Tabela 3. Duração média (dias) dos períodos larval, pupal e longevidade dos adultos de Diatraea saccharalis na dieta padrão e nas cinco dietas acrescidas com corantes. Bandeirantes-PR, 2015.

\begin{tabular}{cccc}
\hline \multirow{2}{*}{ Dieta } & \multicolumn{3}{c}{ Fases de desenvolvimento } \\
\cline { 2 - 4 } & Larval & Pupa & Adulto \\
\hline A & $49,6 \mathrm{ab}^{1}$ & $9,6 \mathrm{a}$ & $6,85 \mathrm{a}$ \\
B & $47,4 \mathrm{a}$ & $10,07 \mathrm{a}$ & $7,33 \mathrm{a}$ \\
\hline C & $47,67 \mathrm{a}$ & $10,64 \mathrm{a}$ & $9 \mathrm{~b}$ \\
D & $53,09 \mathrm{~b}$ & $10,17 \mathrm{a}$ & $5,66 \mathrm{a}$ \\
\hline E & ------ & ------ & ----- \\
\hline F & $55,6 \mathrm{~b}$ & $10,25 \mathrm{a}$ & $9,5 \mathrm{~b}$ \\
\hline
\end{tabular}

19édias seguidas das mesmas letras na coluna não diferem entre si pelo Teste de Tukey (5\%). 
Na dieta acrescida de urucum o número de adultos foi insuficiente para obtenção de posturas. Nos demais tratamentos, o número total de ovos (Tabela 4) apresentou-se inferior aos relatados por PARRA \& MinsFeldt (1992) e Lima (2011) em condições de laboratório. Dentre os tratamentos a testemunha obteve melhor desempenho no número de ovos, mas ainda sim, os baixos valores ressaltam para a questão da adequação de dietas em laboratórios (Veiga et al 2013).

Na testemunha, assim como nos demais tratamentos, obtevese viabilidade total abaixo de $75 \%$ (Tabela 4), que é uma das condições que SingH (1977) e PARRA (2002) descrevem para uma dieta artificial ser adequada. A alta viabilidade total é exigida devido aos custos de criação do inseto, bem como garantir a qualidade destes ao longo das gerações.

Tabela 4. Viabilidade larval (\%), viabilidade pupal (\%) e média do número total de ovos/casal de Diatraea saccharalis. Bandeirantes-PR, 2015.

\begin{tabular}{cccc}
\hline Dieta & $\begin{array}{c}\text { Viab. Larval } \\
\text { (\%) }\end{array}$ & $\begin{array}{c}\text { Viab. Pupal } \\
\text { (\%) }\end{array}$ & No ovos/casal \\
\hline A & $39 \mathrm{ab}^{1}$ & $35 \mathrm{c}$ & $613 \mathrm{c}$ \\
B & $33,5 \mathrm{a}$ & $25 \mathrm{~b}$ & $562 \mathrm{~b}$ \\
\hline C & $49 \mathrm{c}$ & $20 \mathrm{~b}$ & $298^{\mathrm{a}}$ \\
D & $36 \mathrm{ab}$ & $22 \mathrm{~b}$ & $582 \mathrm{~b}$ \\
\hline E & ------ & ----- & ----- \\
\hline F & $30,25 \mathrm{a}$ & $5 \mathrm{a}$ & ----- \\
\hline
\end{tabular}

${ }^{1}$ Médias seguidas das mesmas letras na coluna não diferem entre si pelo Teste de Tukey (5\%).

Apesar do controle biológico da principal praga da cana-deaçúcar Diatraea saccharalis ser reconhecido mundialmente através do parasitoide Cotesia flavipes, pouco se sabe sobre a sua distribuição espacial, o que implica na sua real eficiência quando liberado no campo. Nesse sentido, o uso de marcadores naturais têm se mostrado promissores nos estudos de biologia e comportamento dos insetos, o que justifica a continuidade dos estudos.

\section{AGRADECIMENTOS}

A Fundação Araucária pelo auxílio financeiro e a USIBAN - Açúcar e Álcool Bandeirantes S/A pelo fornecimento de material.

\section{REFERÊNCIAS}

Botelho, P.S.M., 1985. Tabela de vida ecológica e simulação da fase larval da Diatraea saccharalis (Fabr.,1794) (Lepidoptera.: Pyralidae). Tese (Doutorado em Entomologia)- Escola Superior de Agricultura "Luiz de Queiroz", Universidade de São Paulo.110 p.

Castilllo, A., F. Infante, J. Valle \& J.C. Espinoza, 2006. Dispersión del parasitoide africano Phymastichus coffea LaSalle (Hymenoptera: Eulophidae) en un nuevo agroecosistema. Folia Entomologica Mexicana, 45: 319-327.

Fernandes, O. A, 2002. O uso de marcadores no controle biológico, p. 115-123. In: Parra, J. R. P., P. S.M. Botelho, B. S. Corrêa-Ferreira \& J. M. S. BENTO (Eds.). Controle biológico no Brasil: parasitoides e predadores. São Paulo, Manole, 613p.

Gallo, D., O. Nakano, S. Silveira Neto, R. P. L. Carvalho, G. C. Batista, E. Berti Filho, J. R. P. Parra, R. A. Zucchi, S. B. Alves, J. D. Vendramim, L. C. Marchini, J. R. S. Lopes \& C. Omoto, 2002. Entomologia Agrícola. Piracicaba, FEALQ, 920 p.

Hagler, J. R. \& C. G. Jackson, 2001.Methods for marking insects: current techniques and future prospects. Annual Review of Entomology, 46: 511-543.

Hensley, S. D. \& A. H. Hammond, 1968. Laboratory techniques for rearing the sugar cane borer on an artificial diet. Journal of Economic Entomology, 61: 1742-1743.
King, E. G., F. D. Brewer \& D. F. Martin, 1975. Development of Diatraea saccharalis (Lepidoptera: Pyralidae) at constant temperatures. Entomophaga, Paris,20:301-316.

Lima, A. A., 2011. Comparação de dietas artificiais para criação de Diatraea saccharalis (Lepidoptera: Crambidae) e avaliação da qualidade de Cotesia flavipes (Hymenoptera: Braconidae) em criações massais. Dissertação (Mestrado em Sanidade Vegetal : Segurança Alimentar e Ambiental) - Instituto Biológico. 96p.

Mendonça, A. F., 1996. Broca comum da cana-de-açúcar, p.4982. In: Mendonça, A. F. (Ed.). Pragas da cana-de-açúcar. Maceió, Insetos \& Cia., 239p.

Parra, J. R. P., 2001. Técnicas de criação de insetos para programas de controle biológico. Piracicaba, FEALQ, 134p.

Parra, J. R. P., 2002. Criação massal de inimigos naturais, p. 143164. In: Parra, J. R. P., P. S.M. Botelho, B. S. Corrêa-Ferreira \& J. M. S. BENTO (Eds.). Controle biológico no Brasil: parasitoides e predadores. São Paulo, Manole, 613 p.

Parra, J. R. P. \& L. H. Mihsfeldt, 1992. Comparison of artificial diets for rearing the sugarcane borer,p. 195-209. In: Anderson, T. E. \& N. C. Leppla (Eds.).Advances in insect rearing for research and pest management. San Francisco, Westview Press, $519 \mathrm{p}$.

Pinto, A. S., D. E. Nava, M. M. Rossi \& D. T. Malerbo-Souza, 2006. Controle Biológico de Pragas na Prática. Piracicaba, FEALQ, $287 \mathrm{p}$.

StatSoft, 2007. STATISTICA (data analysis software system). Versão 8.o.Disponível em: <www.statsoft.com>.

Singh, P., 1977. Artificial diets for insects, mites and spiders, Plenum, $549 \mathrm{p}$

Southwood, T. R. E., 1978. Ecological methods. London, Chapman and Hall, $391 \mathrm{p}$.

Southwood, T. R. E., 1992. Ecological methods: with particular reference to the study os insect populations. London, Chapman and Hall, 524 p.

Souza, F. P. L. S., E. A. Taguti , M. A. Nouchi \& L. H. Mihsfeldt, 2013. Efeito da beterraba como marcador no desenvolvimento da broca da cana-de-açúcar Diatraea saccharalis (Fabr.). In: Anais do XXII EAIC - Encontro Anual de Iniciação Científica, Foz do Iguaçu, 4 p. Acessível em: $<$ http://200.201.88.178/anais/artigo.php?cod=5607> .

Veiga, A. C. P, A. M. Vacari, H. X. L. Volpe, V. L. Laurentis \& S. A. De Bortoli, 2013.Quality control of Cotesia flavipes (Cameron) (Hymenoptera: Braconidae) from different Brazilian biofactories. Biocontrol Science and Technology, 23: 665-673.

Vilarinho, E. C., 2007. Marcação de Diatraea saccharalis (Fabr.) (Lepidoptera: Crambidae) e dispersão de Spodoptera frugiperda (J. E. Smith)(Lepidoptera: Noctuidae). Tese (Doutorado em Entomologia Agrícola) - Universidade Estadual Paulista "Júlio de Mesquita Filho", Faculdade de Ciências Agrárias e Veterinárias. 59 p.

Volpe, H. X. L., 2009. Distribuição espacial do parasitismo de Cotesia flavipes (Cameron, 1981) (Hymenoptera: Braconidae) em cana-de-açucar. Dissertação (Mestrado em Agronomia) Universidade Estadual Paulista. 67 p.

\section{Recebido em: 29.xi.2015 Aceito em: 25.ii.2016}


Como citar este artigo:

Rando, J.S.S., L.H. Mihsfeldt, F.P.L. de Souza, F.V. Soares, 2016. Marcação de Diatraea saccharalis (Fabr.) com Diferentes Corantes em Dieta Artificial. EntomoBrasilis, 9 (1): 47-50.

Acessível em: doi:10.12741/ebrasilis.v9i1.576
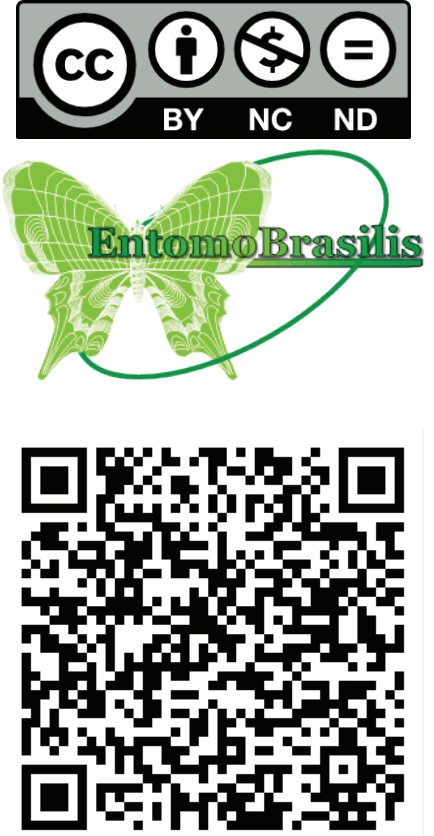